\title{
Asymptotic Stability of Caputo Type Fractional Neutral Dynamical Systems with Multiple Discrete Delays
}

\author{
Hai Zhang, ${ }^{1,2}$ Daiyong $\mathrm{Wu}^{2}$ and Jinde Cao ${ }^{1,3}$ \\ ${ }^{1}$ Department of Mathematics, Research Center for Complex Systems and Network Sciences, Southeast University, \\ Nanjing, Jiangsu 210096, China \\ ${ }^{2}$ Department of Mathematics, Anqing Normal University, Anqing, Anhui 246133, China \\ ${ }^{3}$ Department of Mathematics, Faculty of Science, King Abdulaziz University, Jeddah 21589, Saudi Arabia
}

Correspondence should be addressed to Jinde Cao; jdcao@seu.edu.cn

Received 4 January 2014; Revised 15 June 2014; Accepted 19 June 2014; Published 20 July 2014

Academic Editor: Anna Mercaldo

Copyright (C) 2014 Hai Zhang et al. This is an open access article distributed under the Creative Commons Attribution License, which permits unrestricted use, distribution, and reproduction in any medium, provided the original work is properly cited.

We discuss the delay-independent asymptotic stability of Caputo type fractional-order neutral differential systems with multiple discrete delays. Based on the algebraic approach and matrix theory, the sufficient conditions are derived to ensure the asymptotic stability for all time-delay parameters. By applying the stability criteria, one can avoid solving the roots of transcendental equations. The results obtained are computationally flexible and convenient. Moreover, an example is provided to illustrate the effectiveness and applicability of the proposed theoretical results.

\section{Introduction}

Fractional calculus is regarded as a generalization of the classical integer-order calculus to arbitrary order. Since the fractional-order derivative has nonlocal property and weakly singular kernels, it provides an excellent tool for the description of memory and hereditary properties of dynamical processes. Recently, it has gained increasing interests from researchers in various areas and has become one of the central subjects [1-12]. For more details on fractional calculus theory, one can see the monographs of Miller and Ross [1], Podlubny [2], Kilbas et al. [3], and Diethelm [4]. Lakshmikantham et al. [5] and Baleanu et al. [6] have elaborated the theory of fractional-order dynamics systems.

Stability is an important performance metric for dynamic systems. Meanwhile, time delay has an important effect on the stability and performance of dynamic systems. In the past few decades, numerous results on the stability problem of integer-order delay differential systems have been obtained (see [13-20] and references therein). Recently, there are some results on the stability of fractional-order differential systems [21-33]. For example, Matignon [23, 24] discussed the asymptotic stability of linear fractional-order autonomous systems. In terms of comparison principle [25, 26] and Lyapunov direct method [27], Li et al. [25, 27] obtained the Mittag-Leffler stability theorems of fractionalorder systems. Linear matrix inequality (LMI) method [28] and variational Lyapunov method [29] were also used to investigate the stability of fractional-order systems. Wang et al. [30] investigated Hyers-Ulam-Rassias stability of a certain fractional differential equation by means of the fixed point theorem. Moreover, Rivero et al. [31], Li and Zhang [32], and Choi et al. [33] summarized and reviewed the developments and advances in stability of fractional-order dynamical systems, respectively.

It is worth pointing out that the notable contributions have been made to the stability of fractional-order delay differential systems [34-44]. Many methods have been applied to discuss various types of stability problems for fractional-order delay dynamical systems, such as Gronwall integral inequality method [34-36], final-value theorem of Laplace transform [37], Lyapunov functional method [38, 39], analytical and numerical methods [40], fixed point theorems [41-43], and semigroup theory [42]. 
In this paper, motivated by the aforementioned works, we are devoted to discussing the delay-independent asymptotic stability for linear Caputo fractional neutral differential difference system with multiple discrete delays as follows:

$$
\begin{gathered}
{ }^{C} D^{\alpha}\left[x(t)-\sum_{i=1}^{m} C_{i} x\left(t-\tau_{i}\right)\right]=A x(t)+\sum_{i=1}^{m} B_{i} x\left(t-\tau_{i}\right), \\
t \geq 0, \\
x(t)=\varphi(t), \quad t \in[-\tau, 0],
\end{gathered}
$$

where ${ }^{C} D^{\alpha} x(t)$ denotes an $\alpha$ order Caputo fractional derivative of $x(t), 0<\alpha<1, A, B_{i}, C_{i}$ are $n \times n$ constant matrices, $\tau_{i}(i=1,2, \ldots, m)$ are real constants with $0<$ $\tau_{1} \leq \tau_{2} \leq \cdots \leq \tau_{m}=\tau$, the initial function $\varphi \in$ $\mathbf{C}^{1}\left([-\tau, 0], \mathbb{R}^{n}\right), \quad$ and $\mathbf{C}^{1}\left([-\tau, 0], \mathbb{R}^{n}\right)$ denotes space of continuously differentiable functions mapping the interval $[-\tau, 0]$ into $\mathbb{R}^{n}$.

Compared to integer-order differential systems, the research on the stability of fractional dynamical systems is still at the stage of exploiting and developing. Different from the methods in [34-43], we apply the algebraic approach and matrix theory to establish the delay-independent asymptotic stability criteria for system (1), which do not contain information on delays. We establish the sufficient conditions which ensure that all the roots of characteristic equation lie in open left-half complex plane and are uniformly bounded away from the imaginary axis. At the same time, by applying these stability criteria, one can avoid solving the roots of the transcendental equations. The results obtained are computationally flexible and efficient.

The rest of this paper is organized as follows. In Section 2, we present some definitions, notations, and lemmas related to the main results. In Section 3, the sufficient conditions of the delay-independent asymptotic stability for system (1) are derived based on the algebraic approach and matrix theory. In Section 4, an example is provided to illustrate the effectiveness and applicability of the proposed criteria. Finally, some concluding remarks are drawn in Section 5.

\section{Preliminaries}

In this section, we present some definitions of fractional calculus (see [1-4]), notations, and lemmas used in the paper.

For the sake of convenience, some notations are introduced firstly. Throughout this paper, $\operatorname{det}(A)$ represents the determinant of matrix $A, \sigma(A)$ denotes the spectrum of matrix $A, \rho[A]$ represents the spectral radius of matrix $A$, and $\arg (\sigma(A))$ stands for the principal argument of $\sigma(A)$ defined on $(-\pi, \pi]$.

(a) Riemann-Liouville's fractional integral of order $q>$ 0 for a function $f: \mathbb{R}^{+} \rightarrow \mathbb{R}^{n}$ is given by

$$
D^{-q} f(t)=\frac{1}{\Gamma(q)} \int_{0}^{t}(t-s)^{q-1} f(s) d s, \quad t>0,
$$

where $\Gamma(\cdot)$ is Euler's gamma function. (b) Riemann-Liouville's fractional derivative of order $q$ for a function $f: \mathbb{R}^{+} \rightarrow \mathbb{R}^{n}$ is given by

$$
{ }^{\mathrm{RL}} D^{q} f(t)=\frac{1}{\Gamma(m-q)} \frac{d^{m}}{d t^{m}} \int_{0}^{t}(t-s)^{m-q-1} f(s) d s,
$$

where $0 \leq m-1 \leq q<m, m \in \mathbb{Z}^{+}$.

(c) Caputo's fractional derivative of order $q$ for a function $f: \mathbb{R}^{+} \rightarrow \mathbb{R}^{n}$ is defined as

$$
{ }^{C} D^{q} f(t)=\frac{1}{\Gamma(m-q)} \int_{0}^{t}(t-s)^{m-q-1} f^{(m)}(s) d s,
$$

where $0 \leq m-1 \leq q<m, m \in \mathbb{Z}^{+}$. Here, ${ }^{C} D^{q}$ is still written as $D^{q}$.

(d) The Laplace transform of a function $f(t)$ is defined as

$$
F(s)=\mathfrak{E}[f(t)]=\int_{0}^{+\infty} e^{-s t} f(t) d t, \quad s \in \mathbb{C},
$$

where $\mathbb{C}$ denotes the complex plane and $f(t)$ is $n$ dimensional vector-valued function. For $m-1 \leq q<$ $m$, it follows from [1-4] that

$$
\mathfrak{E}\left[D^{q} f(t)\right]=s^{q} \mathfrak{E}[f(t)]-\sum_{k=0}^{m-1} s^{q-k-1} f^{(k)}(0) .
$$

The following Mittag-Leffler function plays an important role in the study on fractional-order differential systems, which is considered as a natural generalization of the exponential function.

(e) The Mittag-Leffler function in two parameters is defined as

$E_{q, \beta}(z)=\sum_{k=0}^{+\infty} \frac{z^{k}}{\Gamma(k q+\beta)}, \quad q>0, \beta>0, z \in \mathbb{C}$.

In particular, for $\beta=1$, the Mittag-Leffler function in one parameter is given by

$$
E_{q}(z):=E_{q, 1}(z)=\sum_{k=0}^{+\infty} \frac{z^{k}}{\Gamma(1+k q)}, \quad q>0, z \in \mathbb{C} .
$$

Applying the method of steps [34], we obtain the following lemma which generalizes well-known results of integerorder delay differential systems [13] to fractional-order neutral differential systems.

Lemma 1. For system (1), there exists a unique continuous solution on $[0,+\infty)$.

Proof. For system (1), on the interval $[-\tau, 0], x(t)=\varphi(t)$. Thus, when $t \in\left[0, \tau_{1}\right]$, system (1) is given by

$$
D^{\alpha} x(t)=A x(t)+\sum_{i=1}^{m} B_{i} \varphi\left(t-\tau_{i}\right)+D^{\alpha}\left[\sum_{i=1}^{m} C_{i} \varphi\left(t-\tau_{i}\right)\right] .
$$


Since $\sum_{i=1}^{m} B_{i} \varphi\left(t-\tau_{i}\right)+D^{\alpha}\left[\sum_{i=1}^{m} C_{i} \varphi\left(t-\tau_{i}\right)\right]$ is continuous on $\left[0, \tau_{1}\right]$, from [3], we know that there is a unique continuous solution for system (1) on $\left[-\tau, \tau_{1}\right]$, which is denoted as $x_{1}(t), t \in\left[-\tau, \tau_{1}\right]$. Furthermore, $x_{1}(t)$ can be expressed by the following form:

$$
\begin{aligned}
& x_{1}(t) \\
& =\left\{\begin{aligned}
\varphi(t), & t \in[-\tau, 0], \\
E_{\alpha}\left(A t^{\alpha}\right) \varphi(0) & \\
+\int_{0}^{t}(t-\theta)^{\alpha-1} E_{\alpha, \alpha}\left[A(t-\theta)^{\alpha}\right] & \\
& \times\left\{\sum_{i=1}^{m} B_{i} \varphi\left(\theta-\tau_{i}\right)\right. \\
\left.+D^{\alpha}\left[\sum_{i=1}^{m} C_{i} \varphi\left(\theta-\tau_{i}\right)\right]\right\} d \theta, & t \in\left[0, \tau_{1}\right] .
\end{aligned}\right.
\end{aligned}
$$

For $t \in\left[\tau_{1}, 2 \tau_{1}\right]$, system (1) is given by

$$
D^{\alpha} x(t)=A x(t)+\sum_{i=1}^{m} B_{i} x_{1}\left(t-\tau_{i}\right)+D^{\alpha}\left[\sum_{i=1}^{m} C_{i} x_{1}\left(t-\tau_{i}\right)\right] \text {. }
$$

Similarly, since $\sum_{i=1}^{m} B_{i} x_{1}\left(t-\tau_{i}\right)+D^{\alpha}\left[\sum_{i=1}^{m} C_{i} x_{1}\left(t-\tau_{i}\right)\right]$ is continuous on $\left[-\tau, 2 \tau_{1}\right]$, we obtain that $x_{2}(t)$ is a unique continuous solution of system (1) on $\left[-\tau, 2 \tau_{1}\right]$ and

$$
\begin{aligned}
& x_{2}(t) \\
& =\left\{\begin{aligned}
x_{1}(t), & t \in\left[-\tau, \tau_{1}\right], \\
E_{\alpha}\left(A t^{\alpha}\right) \varphi(0) & \\
+\int_{0}^{t}(t-\theta)^{\alpha-1} E_{\alpha, \alpha}\left[A(t-\theta)^{\alpha}\right] & \\
& \times\left\{\sum_{i=1}^{m} B_{i} x_{1}\left(\theta-\tau_{i}\right)\right. \\
\left.+D^{\alpha}\left[\sum_{i=1}^{m} C_{i} x_{1}\left(\theta-\tau_{i}\right)\right]\right\} d \theta, & t \in\left[\tau_{1}, 2 \tau_{1}\right] .
\end{aligned}\right.
\end{aligned}
$$

Assume that system (1) has a unique solution $x_{k}(t)$ on $\left[(k-1) \tau_{1}, k \tau_{1}\right]$. For $t \in\left[k \tau_{1},(k+1) \tau_{1}\right]$, system (1) is given by

$$
D^{\alpha} x(t)=A x(t)+\sum_{i=1}^{m} B_{i} x_{k}\left(t-\tau_{i}\right)+D^{\alpha}\left[\sum_{i=1}^{m} C_{i} x_{k}\left(t-\tau_{i}\right)\right] \text {. }
$$

Similarly, since $\sum_{i=1}^{m} B_{i} x_{k}\left(t-\tau_{i}\right)+D^{\alpha}\left[\sum_{i=1}^{m} C_{i} x_{k}\left(t-\tau_{i}\right)\right]$ is continuous on $[k \tau,(k+1) \tau]$, we obtain that $x_{k+1}(t)$ is a unique continuous solution of system (1) on $\left[k \tau_{1},(k+1) \tau_{1}\right]$ and

$$
\begin{aligned}
& x_{k+1}(t) \\
& =\left\{\begin{array}{cc}
x_{k}(t), & t \in\left[-\tau, k \tau_{1}\right], \\
E_{\alpha}\left(A t^{\alpha}\right) \varphi(0) & \\
+\int_{0}^{t}(t-\theta)^{\alpha-1} E_{\alpha, \alpha}\left[A(t-\theta)^{\alpha}\right] & \\
& \times\left\{\sum_{i=1}^{m} B_{i} x_{k}\left(\theta-\tau_{i}\right)\right. \\
\left.+D^{\alpha}\left[\sum_{i=1}^{m} C_{i} x_{k}\left(\theta-\tau_{i}\right)\right]\right\} d \theta, & t \in\left[k \tau_{1},(k+1) \tau_{1}\right] .
\end{array}\right.
\end{aligned}
$$

According to the mathematical induction, we know that system (1) has a unique continuous solution on $\left[0, k \tau_{1}\right], k=$ $1,2, \ldots$.

Now, for any $T>0$, we assert that system (1) has a unique continuous solution on $[0, T]$. In fact, three cases are discussed as follows.

Case 1. When $(k+1) \tau_{1}=T$, we know that the assertion is true.

Case 2. When $0<T-(k+1) \tau_{1}<\tau_{1}$, we only need to prove that system (1) has a unique continuous solution on $\left[\tau_{1}, T\right]$. For $t \in\left[\tau_{1}, T\right]$, we denote $t_{1}=t-\tau_{1} \in\left[0, T-\tau_{1}\right] \subset$ $\left[0,(k+1) \tau_{1}\right]$, and we can use the similar proof to obtain the conclusion.

Case 3. When $T-(k+1) \tau_{1}>\tau_{1}$, we can repeat the above process until the condition of Case 2 is satisfied.

Note that $T$ is an arbitrary positive real number; then, we know that system (1) has a unique continuous solution on $[0,+\infty)$. Therefore, the proof is completed.

Remark 2. Lemma 1 ensures the existence and uniqueness of solution for system (1) on $[0,+\infty)$. Evidently, Caputo's fractional derivative of a constant is equal to zero; then, $x(t) \equiv$ 0 is the zero solution of system (1).

Definition 3. The zero solution $x(t) \equiv 0$ of system (1) is called delay-independent globally asymptotically stable if, for any initial function $\varphi(\cdot) \in \mathbf{C}^{1}\left([-\tau, 0], \mathbb{R}^{n}\right)$, its analytic solution $x(t)$ satisfies $\lim _{t \rightarrow+\infty} x(t)=0$ for all the time delays $0<\tau_{1} \leq \tau_{2} \leq \cdots \leq \tau_{m}$.

Next, we discuss the characteristic equation and delayindependent globally asymptotic stability of system (1).

From [1-4], the Laplace transform of Caputo fractionalorder derivative $D^{\alpha} f(t)$ is given as follows:

$$
\mathfrak{E}\left[D^{\alpha} f(t)\right]=s^{\alpha} \mathfrak{E}[f(t)]-s^{\alpha-1} f(0), \quad 0<\alpha<1 .
$$

Applying the Laplace transform on both sides of system (1) yields

$$
\begin{aligned}
& s^{\alpha} £\left[x(t)-\sum_{i=1}^{m} C_{i} x\left(t-\tau_{i}\right)\right]-s^{\alpha-1} \varphi(0)+s^{\alpha-1} \sum_{i=1}^{m} C_{i} \varphi\left(-\tau_{i}\right) \\
& =A £[x(t)]+\sum_{i=1}^{m} B_{i} £\left[x\left(t-\tau_{i}\right)\right] .
\end{aligned}
$$

Note that

$$
\begin{array}{r}
£\left[x\left(t-\tau_{i}\right)\right]=e^{-s \tau_{i}} \mathfrak{E}[x(t)]+e^{-s \tau_{i}} \int_{-\tau_{i}}^{0} e^{-s t} \varphi(t) d t, \\
i=1,2, \ldots, m ;
\end{array}
$$


thus, we obtain

$$
\begin{aligned}
\Delta\left(s, \tau_{i}\right) £[x(t)]= & s^{\alpha-1} \varphi(0)-s^{\alpha-1} \sum_{i=1}^{m} C_{i} \varphi\left(-\tau_{i}\right) \\
& +\sum_{i=1}^{m} B_{i} e^{-s \tau_{i}} \int_{-\tau_{i}}^{0} e^{-s t} \varphi(t) d t \\
& +s^{\alpha} \sum_{i=1}^{m} C_{i} e^{-s \tau_{i}} \int_{-\tau_{i}}^{0} e^{-s t} \varphi(t) d t,
\end{aligned}
$$

where

$$
\Delta\left(s, \tau_{i}\right)=\left[s^{\alpha} I-A-\sum_{i=1}^{m} B_{i} e^{-s \tau_{i}}-s^{\alpha} \sum_{i=1}^{m} C_{i} e^{-s \tau_{i}}\right]
$$

is the characteristic matrix of system (1). Multiplying $s$ on both sides of (18) yields

$$
\begin{aligned}
\Delta\left(s, \tau_{i}\right)\{s \mathfrak{E}[x(t)]\}=s\left\{s^{\alpha-1} \varphi(0)-s^{\alpha-1} \sum_{i=1}^{m} C_{i} \varphi\left(-\tau_{i}\right)\right. \\
+\sum_{i=1}^{m} B_{i} e^{-s \tau_{i}} \int_{-\tau_{i}}^{0} e^{-s t} \varphi(t) d t \\
\left.+s^{\alpha} \sum_{i=1}^{m} C_{i} e^{-s \tau_{i}} \int_{-\tau_{i}}^{0} e^{-s t} \varphi(t) d t\right\} .
\end{aligned}
$$

By means of the final-value theorem of Laplace transform [45] and Definition 3, if all the roots of characteristic equation $\operatorname{det}\left[\Delta\left(s, \tau_{i}\right)\right]=0$ lie in open left-half complex plane and are uniformly bounded away from the imaginary axis, then the zero solution of system (1) is delay-independent globally asymptotically stable.

Therefore, we immediately have the following conclusion.

Lemma 4. If all the roots of characteristic equation

$$
\operatorname{det}\left[s^{\alpha} I-A-\sum_{i=1}^{m} B_{i} e^{-s \tau_{i}}-s^{\alpha} \sum_{i=1}^{m} C_{i} e^{-s \tau_{i}}\right]=0
$$

lie in open left-half complex plane and are uniformly bounded away from the imaginary axis, then the zero solution of system (1) is delay-independent globally asymptotically stable.

Remark 5. As we know, when $\alpha=1$ and $\tau_{1}=\tau_{2}=\cdots=$ $\tau_{m}=0$, the characteristic equation

$$
\operatorname{det}\left[\lambda I-A-\sum_{i=1}^{m}\left(B_{i}+\lambda C_{i}\right)\right]=0
$$

is an algebraic equation of $\lambda$, and (22) only has $n$ roots distributed in the complex plane. However, the characteristic equation $\operatorname{det}\left[\Delta\left(s, \tau_{i}\right)\right]=0$ has countably infinite roots with $\alpha=1$ and some $\tau_{i}>0$ (see [13]). For $0<\alpha<$ 1 and $\tau_{i}>0(i=1,2, \ldots, m)$, it is very difficult to solve the roots of the transcendental equation (21) in practice. Based on these considerations, we are devoted to establishing the algebraic stability criteria of system (1) in the next section.

\section{Stability Criteria for System (1)}

In this section, we derive the sufficient conditions of delayindependent globally asymptotic stability for system (1). Applying the algebraic method, we investigate the distribution of roots for equation $\operatorname{det}\left[\Delta\left(s, \tau_{i}\right)\right]=0$ in any neighborhood of the infinity and find a positive number $\delta>0$ such that any characteristic root $s$ satisfies $\mathfrak{R} e(s)<-\delta<0$, where $\mathfrak{R} e(s)$ represents the real part of the complex number $s$.

Theorem 6. The zero solution $x(t) \equiv 0$ of system (1) is delayindependent globally asymptotically stable if the following conditions are satisfied:

$$
\begin{aligned}
& \left(H_{1}\right) \quad|\arg (\sigma(A))|>\frac{\alpha \pi}{2}, \\
& \left(H_{2}\right) \quad \rho\left[\sum_{i=1}^{m} C_{i}\right]<1, \\
& \left(H_{3}\right) \quad \sup _{\Re(s) \geq 0} \rho\left[\left(s^{\alpha} I-A\right)^{-1} \sum_{i=1}^{m}\left(B_{i}+s^{\alpha} C_{i}\right)\right]<1 .
\end{aligned}
$$

Proof. Note that $|\arg (\sigma(A))|>\alpha \pi / 2$; then, all the roots of equation $\operatorname{det}(\lambda I-A)=0$ satisfy $|\arg (\lambda)|>\alpha \pi / 2$. Let $s^{\alpha}=\lambda$; then, all the roots of equation $\operatorname{det}\left(s^{\alpha} I-A\right)=$ 0 satisfy $|\arg (s)|>\pi / 2$; that is, $\mathfrak{R} e(s)<0$. Therefore, matrix $A$ is invertible and $\left(s^{\alpha} I-A\right)^{-1}$ is well defined when $|\arg (\sigma(A))|>\alpha \pi / 2$ and $\mathfrak{R} e(s) \geq 0$.

For $\mathfrak{R} e(s) \geq 0$, it follows from the characteristic polynomial of system (1) that

$$
\begin{aligned}
& \operatorname{det} \Delta\left(s, \tau_{i}\right) \\
& =\operatorname{det}\left[s^{\alpha} I-A-\sum_{i=1}^{m} B_{i} e^{-s \tau_{i}}-s^{\alpha} \sum_{i=1}^{m} C_{i} e^{-s \tau_{i}}\right] \\
& =\operatorname{det}\left[s^{\alpha} I-A\right] \operatorname{det}\left[I-\left(s^{\alpha} I-A\right)^{-1}\right. \\
& \left.=\operatorname{det}\left[s^{\alpha} I-A\right] \quad \times \sum_{i=1}^{m}\left(B_{i}+s^{\alpha} C_{i}\right) e^{-s \tau_{i}}\right] \\
& \quad \times \prod_{j=1}^{n}\left(1-\lambda_{j}\left[\left(s^{\alpha} I-A\right)^{-1} \sum_{i=1}^{m}\left(B_{i}+s^{\alpha} C_{i}\right) e^{-s \tau_{i}}\right]\right) .
\end{aligned}
$$

Then, we have

$$
\begin{array}{r}
\left|\lambda_{j}\left[\left(s^{\alpha} I-A\right)^{-1} \sum_{i=1}^{m}\left(B_{i}+s^{\alpha} C_{i}\right) e^{-s \tau_{i}}\right]\right| \\
\leq \rho\left[\left(s^{\alpha} I-A\right)^{-1} \sum_{i=1}^{m}\left(B_{i}+s^{\alpha} C_{i}\right)\right]\left|e^{-s \tau}\right|<1, \\
j=1,2, \ldots, n .
\end{array}
$$

Combining (24) and (25), we have $\operatorname{det} \Delta\left(s, \tau_{i}\right) \neq 0$ for $\mathfrak{R} e(s) \geq 0$ and $\tau_{i}>0, i=1,2, \ldots, m$; that is, if, 
conditions $\left(H_{1}\right)$ and $\left(H_{3}\right)$ are satisfied, then the characteristic equation $(21)$ implies that $\mathfrak{R} e(s)<0$.

Suppose that there exists a sequence of roots $\left\{s_{n}\right\}$ of the characteristic equation (21) whose real parts are not uniformly bounded away from zero; that is, $\mathfrak{R} e\left(s_{n}\right)<$ 0 and $\mathfrak{R e}\left(s_{n}\right) \rightarrow 0$ as $n \rightarrow+\infty$. Note that any eigenvalue $\lambda_{j}\left[\left(s^{\alpha} I-A\right)^{-1} \sum_{i=1}^{m}\left(B_{i}+s^{\alpha} C_{i}\right)\right]$ is a continuous function of $s$ for $\mathfrak{R} e(s) \geq 0$; then, it follows from $\left(\mathrm{H}_{2}\right)$ that

$$
\begin{array}{r}
\rho\left[\left(s^{\alpha} I-A\right)^{-1} \sum_{i=1}^{m}\left(B_{i}+s^{\alpha} C_{i}\right)\right] \longrightarrow \rho\left[\sum_{i=1}^{m} C_{i}\right]<1, \\
s \longrightarrow+\infty .
\end{array}
$$

Hence, $\left|\lambda_{j}\left[\left(s^{\alpha} I-A\right)^{-1} \sum_{i=1}^{m}\left(B_{i}+s^{\alpha} C_{i}\right)\right]\right|$ reach the maximum value for $\mathfrak{R} e(s) \geq 0$. From condition $\left(\mathrm{H}_{3}\right)$, there exists a positive constant $\varepsilon$ such that

$$
\begin{aligned}
& \sup _{\Re e(s) \geq 0} \rho\left[\left(s^{\alpha} I-A\right)^{-1} \sum_{i=1}^{m}\left(B_{i}+s^{\alpha} C_{i}\right)\right] \\
& =\sup _{\Re e(s) \geq 0} \max _{1 \leq j \leq n}\left|\lambda_{j}\left[\left(s^{\alpha} I-A\right)^{-1} \sum_{i=1}^{m}\left(B_{i}+s^{\alpha} C_{i}\right)\right]\right|=1-\varepsilon .
\end{aligned}
$$

Then, equality (27) implies that

$$
\sup _{\operatorname{Re}(\zeta)=0} \rho\left[\left(\zeta^{\alpha} I-A\right)^{-1} \sum_{i=1}^{m}\left(B_{i}+\zeta^{\alpha} C_{i}\right)\right] \leq 1-\varepsilon
$$

When the positive integer $n$ is large enough, there exist a positive constant $\varepsilon^{*}\left(0<\varepsilon^{*}<\varepsilon\right)$ and a characteristic root $s_{n}$ such that $\left|\mathfrak{R} e\left(s_{n}\right)\right|$ is sufficiently small, $\mathfrak{R} e\left(s_{n}\right)<$ 0 and

$$
\begin{aligned}
& \mid \max _{1 \leq j \leq n} \lambda_{j}\left[\left(s_{n}^{\alpha} I-A\right)^{-1} \sum_{i=1}^{m}\left(B_{i}+s_{n}^{\alpha} C_{i}\right)\right] \\
& -\sup _{\mathfrak{R e}(\zeta)=0} \rho\left[\left(\zeta^{\alpha} I-A\right)^{-1} \sum_{i=1}^{m}\left(B_{i}+\zeta^{\alpha} C_{i}\right)\right] \mid<\varepsilon^{*} .
\end{aligned}
$$

For $\Re e(\zeta)=0$, from (28) and (29), we have

$$
\begin{aligned}
\mid \lambda_{j} & {\left[\left(s_{n}^{\alpha} I-A\right)^{-1} \sum_{i=1}^{m}\left(B_{i}+s_{n}^{\alpha} C_{i}\right)\right] \mid } \\
& \leq \sup _{\Re e(\zeta)=0} \rho\left[\left(\zeta^{\alpha} I-A\right)^{-1} \sum_{i=1}^{m}\left(B_{i}+\zeta^{\alpha} C_{i}\right)\right]+\varepsilon^{*} \\
& \leq 1-\varepsilon+\varepsilon^{*}<1, \quad j=1,2, \ldots, n .
\end{aligned}
$$

Choosing $n$ large enough yields

$$
\left|\lambda_{j}\left[\left(s_{n}^{\alpha} I-A\right)^{-1} \sum_{i=1}^{m}\left(B_{i}+s_{n}^{\alpha} C_{i}\right) e^{-s \tau_{i}}\right]\right|<1, \quad j=1,2, \ldots, n .
$$

Therefore, for $\mathfrak{R} e\left(s_{n}\right)<0$ and $\mathfrak{R} e\left(s_{n}\right) \rightarrow 0$ as $n \rightarrow+\infty$, one can obtain

$$
\begin{aligned}
\operatorname{det} \Delta\left(s_{n}, \tau_{i}\right)=\operatorname{det} & {\left[s_{n}^{\alpha} I-A-\sum_{i=1}^{m} B_{i} e^{-s_{n} \tau_{i}}\right.} \\
& \left.-s_{n}^{\alpha} \sum_{i=1}^{m} C_{i} e^{-s_{n} \tau_{i}}\right] \neq 0,
\end{aligned}
$$

which contradicts the assumption that $\left\{s_{n}\right\}$ is a sequence of roots of the characteristic equation (21). In view of Lemma 4, the proof is completed.

Theorem 7. The zero solution $x(t) \equiv 0$ of system (1) is delayindependent globally asymptotically stable if the following conditions are satisfied:

$$
\begin{aligned}
& \left(H_{1}\right) \quad|\arg (\sigma(A))|>\frac{\alpha \pi}{2}, \quad 0<\alpha<1, \\
& \left(H_{4}\right) \quad \rho\left[\sum_{i=1}^{m} \xi_{i} C_{i}\right]<1, \quad \xi_{i} \in \mathbb{C},\left|\xi_{i}\right| \leq 1, i=1,2, \ldots, m, \\
& \left(H_{5}\right) \quad \rho\left[\left(s^{\alpha} I-A\right)^{-1} \sum_{i=1}^{m}\left(B_{i}+s^{\alpha} C_{i}\right) \xi_{i}\right]<1,
\end{aligned}
$$$$
\Re(s)=0, \quad \xi_{i} \in \mathbb{C}, \quad\left|\xi_{i}\right|=1, \quad i=1,2, \ldots, m \text {. }
$$

Proof. According to condition $\left(H_{1}\right)$ and the proof of Theorem 6, we know that matrix $A$ is invertible and $\left(s^{\alpha} I-A\right)^{-1}$ is well defined when $|\arg (\sigma(A))|>$ $\alpha \pi / 2$ and $\mathfrak{R} e(s)=0$.

On the one hand, any eigenvalue $\lambda\left[\left(s^{\alpha} I-A\right)^{-1}\right.$ $\left.\left(\sum_{i=1}^{m} \xi_{i} B_{i}+s^{\alpha} \sum_{i=1}^{m} \xi_{i} C_{i}\right)\right]$ is an algebraic function of $\left(\xi_{1}, \xi_{2}, \ldots, \xi_{m}\right)$ with $\left|\xi_{i}\right|<1$ and is continuous with $\left|\xi_{i}\right| \leq 1, i=1,2, \ldots, m$. An application of the maximum modulus principle yields that

$$
\begin{array}{r}
\rho\left[\left(s^{\alpha} I-A\right)^{-1}\left(\sum_{i=1}^{m} \xi_{i} B_{i}+s^{\alpha} \sum_{i=1}^{m} \xi_{i} C_{i}\right)\right]<1 \\
\mathfrak{R} e(s)=0, \quad \xi_{i} \in \mathbb{C}, \quad\left|\xi_{i}\right|=1
\end{array}
$$

is equivalent to

$$
\begin{array}{r}
\rho\left[\left(s^{\alpha} I-A\right)^{-1}\left(\sum_{i=1}^{m} \xi_{i} B_{i}+s^{\alpha} \sum_{i=1}^{m} \xi_{i} C_{i}\right)\right]<1, \\
\mathfrak{R} e(s)=0, \quad \xi_{i} \in \mathbb{C}, \quad\left|\xi_{i}\right| \leq 1 .
\end{array}
$$

In view of the expression of $\left(s^{\alpha} I-A\right)^{-1}\left(\sum_{i=1}^{m} \xi_{i} B_{i}+\right.$ $\left.s^{\alpha} \sum_{i=1}^{m} \xi_{i} C_{i}\right)$, as $\mathfrak{R} e(s)=0$ and $s \rightarrow \infty$, one can obtain

$$
\begin{aligned}
\rho\left[\left(s^{\alpha} I-A\right)^{-1}\left(\sum_{i=1}^{m} \xi_{i} B_{i}+s^{\alpha} \sum_{i=1}^{m} \xi_{i} C_{i}\right)\right] & \longrightarrow \rho\left[\sum_{i=1}^{m} \xi_{i} C_{i}\right]<1, \\
& \forall \xi_{i} \in \mathbb{C}, \quad\left|\xi_{i}\right| \leq 1 .
\end{aligned}
$$


It follows from (36) that

$$
\begin{array}{r}
\sup _{\Re(s)=0} \rho\left[\left(s^{\alpha} I-A\right)^{-1}\left(\sum_{i=1}^{m} \xi_{i} B_{i}+s^{\alpha} \sum_{i=1}^{m} \xi_{i} C_{i}\right)\right] \leq 1, \\
\forall \xi_{i} \in \mathbb{C}, \quad\left|\xi_{i}\right| \leq 1 .
\end{array}
$$

Applying the maximum modulus principle on the unbounded region $\{s \mid s \in \mathbb{C}, \mathfrak{R} e(s) \geq 0\}$, then (35) implies that

$$
\begin{array}{r}
\rho\left[\left(s^{\alpha} I-A\right)^{-1}\left(\sum_{i=1}^{m} \xi_{i} B_{i}+s^{\alpha} \sum_{i=1}^{m} \xi_{i} C_{i}\right)\right]<1, \\
\mathfrak{R} e(s) \geq 0, \quad \xi_{i} \in \mathbb{C}, \quad\left|\xi_{i}\right| \leq 1 .
\end{array}
$$

On the other hand, suppose that there exists some $s_{0} \in$ $\{s \mid s \in \mathbb{C}, \mathfrak{R} e(s) \geq 0\}$ such that

$$
\begin{array}{r}
\operatorname{det}\left[s_{0}^{\alpha} I-A-\sum_{i=1}^{m} B_{i} e^{-s_{0} \tau_{i}}-s_{0}^{\alpha} \sum_{i=1}^{m} C_{i} e^{-s_{0} \tau_{i}}\right]=0, \\
s_{0} \in\{s \mid s \in \mathbb{C}, \mathfrak{R} e(s) \geq 0\} .
\end{array}
$$

Choose $\xi_{i}^{0}=e^{-s_{0} \tau_{i}}$ such that $\left|\xi_{i}^{0}\right| \leq 1, i=1,2, \ldots, m$. It follows from (39) that

$$
\begin{array}{r}
1 \in \sigma\left[\left(s_{0}^{\alpha} I-A\right)^{-1}\left(\sum_{i=1}^{m} B_{i} e^{-s_{0} \tau_{i}}+s_{0}^{\alpha} \sum_{i=1}^{m} C_{i} e^{-s_{0} \tau_{i}}\right)\right], \\
s_{0} \in\{s \mid s \in \mathbb{C}, \mathfrak{R} e(s) \geq 0\},
\end{array}
$$

which contradicts inequality (38). Therefore, if conditions $\left(H_{1}\right),\left(H_{4}\right)$, and $\left(H_{5}\right)$ are satisfied, then the characteristic equation (21) implies that $\mathfrak{R} e(s)<0$.

The rest of the proof is similar to that of Theorem 6 ; then, the conclusion holds.

According to the proof of Theorem 7, we have the result as follows.

Corollary 8. The zero solution $x(t) \equiv 0$ of system (1) is delayindependent globally asymptotically stable if the following conditions are satisfied:

$$
\begin{aligned}
& \left(H_{1}\right) \quad|\arg (\sigma(A))|>\frac{\alpha \pi}{2}, \quad 0<\alpha<1, \\
& \left(H_{4}\right) \quad \rho\left[\sum_{i=1}^{m} \xi_{i} C_{i}\right]<1, \quad \xi_{i} \in \mathbb{C},\left|\xi_{i}\right| \leq 1, i=1,2, \ldots, m, \\
& \left(H_{6}\right) \quad \rho\left[\left(s^{\alpha} I-A\right)^{-1} \sum_{i=1}^{m}\left(B_{i}+s^{\alpha} C_{i}\right) \xi_{i}\right]<1,
\end{aligned}
$$$$
\mathfrak{R} e(s) \geq 0, \quad \xi_{i} \in \mathbb{C}, \quad\left|\xi_{i}\right| \leq 1, \quad i=1,2, \ldots, m .
$$

Assume that $|\arg (\sigma(A))|>\alpha \pi / 2,0<\alpha<1$. Define the following matrices:

$$
\begin{gathered}
L=(I-A)^{-1} \sum_{i=1}^{m}\left(B_{i}+C_{i}\right), \\
M=(I-A)^{-1} \sum_{i=1}^{m}\left(B_{i}-C_{i}\right), \\
N=(I-A)^{-1}(I+A) .
\end{gathered}
$$

Let

$$
s^{\alpha}=\frac{1-z}{1+z}, \quad \mathfrak{R} e(s) \geq 0,0<\alpha<1 ;
$$

then, we have $|z| \leq 1$.

Theorem 9. The zero solution $x(t) \equiv 0$ of system (1) is delayindependent globally asymptotically stable if the following conditions are satisfied:

$$
\begin{aligned}
& \left(H_{1}\right) \quad|\arg (\sigma(A))|>\frac{\alpha \pi}{2}, \quad 0<\alpha<1, \\
& \left(H_{2}\right) \quad \rho\left[\sum_{i=1}^{m} C_{i}\right]<1, \\
& \left(H_{7}\right) \sup _{|z| \leq 1} \rho\left[(I-z N)^{-1}(L+z M)\right]<1 .
\end{aligned}
$$

Proof. From Theorem 6, we only need to prove that the following equality holds:

$$
\left(s^{\alpha} I-A\right)^{-1} \sum_{i=1}^{m}\left(B_{i}+s^{\alpha} C_{i}\right)=(I-z N)^{-1}(L+z M),
$$

$$
\mathfrak{R} e(s) \geq 0, \quad|z| \leq 1 .
$$

In fact, it is easy to obtain

$$
\begin{aligned}
I-z N= & {\left[I-\frac{1-s^{\alpha}}{1+s^{\alpha}}(I-A)^{-1}(I+A)\right] } \\
= & {\left[\left(1+s^{\alpha}\right) I-\left(1-s^{\alpha}\right)(I-A)^{-1}(I+A)\right]\left(1+s^{\alpha}\right)^{-1} } \\
= & (I-A)^{-1}\left[(I-A)\left(1+s^{\alpha}\right)-\left(1-s^{\alpha}\right)(I+A)\right] \\
& \times\left(1+s^{\alpha}\right)^{-1} \\
= & 2(I-A)^{-1}\left(s^{\alpha} I-A\right)\left(1+s^{\alpha}\right)^{-1} .
\end{aligned}
$$


For $\mathfrak{R} e(s) \geq 0$, it follows from $\left(H_{1}\right)$ and (43) that $|z| \leq 1$; then, matrix $(I-z N)$ is invertible. Hence,

$$
\begin{aligned}
L+z M= & {\left[(I-A)^{-1} \sum_{i=1}^{m}\left(B_{i}+C_{i}\right)\right.} \\
& \left.\quad+\frac{1-s^{\alpha}}{1+s^{\alpha}}(I-A)^{-1} \sum_{i=1}^{m}\left(B_{i}-C_{i}\right)\right] \\
= & (I-A)^{-1}\left[\sum_{i=1}^{m}\left(B_{i}+C_{i}\right)+\frac{1-s^{\alpha}}{1+s^{\alpha}} \sum_{i=1}^{m}\left(B_{i}-C_{i}\right)\right] \\
= & (I-A)^{-1}\left[\sum_{i=1}^{m}\left(B_{i}+C_{i}\right)\left(1+s^{\alpha}\right)\right. \\
& \left.+\left(1-s^{\alpha}\right) \sum_{i=1}^{m}\left(B_{i}-C_{i}\right)\right]\left(1+s^{\alpha}\right)^{-1} \\
= & 2(I-A)^{-1} \sum_{i=1}^{m}\left(B_{i}+s^{\alpha} C_{i}\right)\left(1+s^{\alpha}\right)^{-1} .
\end{aligned}
$$

Combining (46) and (47) yields that (45) holds. Therefore, we complete the proof.

Next, the asymptotic stability criteria for two special cases of system (1) are presented.

(i) When $C_{i}=0, i=1,2, \ldots, m$, system (1) reduces to Caputo fractional-order linear retarded type differential difference systems with multiple delays as follows:

$$
\begin{gathered}
D^{\alpha} x(t)=A x(t)+\sum_{i=1}^{m} B_{i} x\left(t-\tau_{i}\right), \quad t \geq 0, \\
x(t)=\varphi(t), \quad t \in[-\tau, 0],
\end{gathered}
$$

where $D^{\alpha} x(t)$ denotes an $\alpha$ order Caputo fractional derivative of $x(t), 0<\alpha<1, A, B_{i}$ are $n \times n$ constant matrices, $\tau_{i}(i=1,2, \ldots, m)$ are real constants with $0<\tau_{1} \leq \tau_{2} \leq \cdots \leq \tau_{m}=\tau, \varphi \in \mathbf{C}\left([-\tau, 0], \mathbb{R}^{n}\right)$, and $\mathbf{C}\left([-\tau, 0], \mathbb{R}^{n}\right)$ denotes space of continuous functions mapping the interval $[-\tau, 0]$ into $\mathbb{R}^{n}$.

Similar to Lemma 1, if the initial function $\varphi \in$ $\mathbf{C}\left([-\tau, 0], \mathbb{R}^{n}\right)$, then there exists a unique continuous solution for system $(48)$ on $[0,+\infty)$.

Corollary 10. The zero solution $x(t) \equiv 0$ of system (48) is delay-independent globally asymptotically stable if the following conditions are satisfied:

$$
\begin{gathered}
\left(H_{1}\right) \quad|\arg (\sigma(A))|>\frac{\alpha \pi}{2}, \quad 0<\alpha<1, \\
\left(H_{8}\right) \quad \rho\left[\left(s^{\alpha} I-A\right)^{-1} \sum_{i=1}^{m} B_{i} \xi_{i}\right]<1, \\
\mathfrak{R} e(s)=0, \quad \xi_{i} \in \mathbb{C}, \quad\left|\xi_{i}\right|=1 .
\end{gathered}
$$

(ii) When $\tau_{i}=0, i=1,2, \ldots, m$, system (1) reduces to Caputo fractional-order linear autonomous differential systems:

$$
\begin{gathered}
{\left[I-\sum_{i=1}^{m} C_{i}\right] D^{\alpha} x(t)=\left[A+\sum_{i=1}^{m} B_{i}\right] x(t), \quad t \geq 0,} \\
x(0)=x_{0}
\end{gathered}
$$

where $D^{\alpha} x(t)$ denotes an $\alpha$ order Caputo fractional derivative of $x(t), 0<\alpha<1$, and $A, B_{i}, C_{i}$ are $n \times$ $n$ constant matrices.

An application of the results in $[23,24]$ yields the following conclusion.

Corollary 11. The zero solution $x(t) \equiv 0$ of system (50) is globally asymptotically stable if the following conditions are satisfied:

$$
\begin{aligned}
& \left(H_{9}\right) \quad \operatorname{det}\left[I-\sum_{i=1}^{m} C_{i}\right] \neq 0, \\
& \left(H_{10}\right) \quad|\arg (\sigma(G))|>\frac{\alpha \pi}{2}, \\
& G=\left(I-\sum_{i=1}^{m} C_{i}\right)^{-1}\left(A+\sum_{i=1}^{m} B_{i}\right) .
\end{aligned}
$$

Remark 12. When $\operatorname{det}\left[I-\sum_{i=1}^{m} C_{i}\right]=0$, system (50) reduces to linear fractional singular differential system. The stability analysis of linear fractional singular (delay) differential systems will become our future investigative works.

\section{An Illustrative Example}

The following example is presented to illustrate the effectiveness and applicability of the proposed stability criteria.

Example 1. Consider system (1) with

$$
\begin{gathered}
A=\left[\begin{array}{cc}
-4 & 2 \\
4 & -10
\end{array}\right], \quad B_{1}=\left[\begin{array}{cc}
0 & 1 \\
-2 & 3
\end{array}\right], \\
B_{2}=\left[\begin{array}{cc}
3 & -1 \\
2 & 0
\end{array}\right], \quad C_{1}=\left[\begin{array}{cc}
\frac{2}{3} & -\frac{1}{6} \\
\frac{1}{3} & -\frac{1}{6}
\end{array}\right], \\
C_{2}=\left[\begin{array}{rr}
-\frac{1}{6} & \frac{1}{6} \\
-\frac{1}{3} & \frac{2}{3}
\end{array}\right], \quad \alpha=\frac{1}{2}, \\
\tau_{1}=1, \quad \tau_{2}=2, \quad \tau=2 .
\end{gathered}
$$

The initial function is given by $\varphi(t)=t, t \in[-2,0]$. Let

$$
\begin{array}{r}
\Theta=\left(s^{\alpha} I-A\right)^{-1}\left(\xi_{1} B_{1}+\xi_{2} B_{2}+s^{\alpha} \xi_{1} C_{1}+s^{\alpha} \xi_{2} C_{2}\right), \\
\Re e(s)=0, \quad\left|\xi_{1}\right|=\left|\xi_{2}\right|=1 .
\end{array}
$$


By computation, the eigenvalues of matrix $\Theta$ give

$$
\begin{aligned}
& \lambda_{1}(\Theta)=\left(s^{\alpha}+6\right)^{-1}\left(\xi_{1}+2 \xi_{2}+\frac{1}{2} s^{\alpha} \xi_{1}+\frac{1}{3} s^{\alpha} \xi_{2}\right) \\
& \lambda_{2}(\Theta)=\left(s^{\alpha}+8\right)^{-1}\left(2 \xi_{1}+\xi_{2}+\frac{1}{3} s^{\alpha} \xi_{1}+\frac{1}{2} s^{\alpha} \xi_{2}\right)
\end{aligned}
$$

Note that $\sigma(A)=\{-6,-8\}$; then, we have

$$
|\arg (\sigma(A))|=\pi>\frac{\alpha \pi}{2}, \quad \alpha=\frac{1}{2} .
$$

It is not difficult to verify that

$$
\begin{aligned}
& \rho\left[\xi_{1} C_{1}+\xi_{2} C_{2}\right]<1, \quad\left|\xi_{1}\right| \leq 1,\left|\xi_{2}\right| \leq 1, \\
& \rho[\Theta]<1, \quad \Re e(s)=0,\left|\xi_{1}\right|=1,\left|\xi_{2}\right|=1 .
\end{aligned}
$$

Thus, conditions $\left(H_{1}\right),\left(H_{3}\right)$, and $\left(H_{4}\right)$ are satisfied. Therefore, it follows from Theorem 7 that the zero solution $x(t) \equiv$ 0 of system (1) with the coefficient matrices (52) is delayindependent globally asymptotically stable.

In fact, the characteristic equation of system (1) with the coefficient matrices (52) can be expressed as

$$
\operatorname{det}\left[s^{\alpha} I-A-\sum_{i=1}^{m} B_{i} e^{-s \tau_{i}}-s^{\alpha} \sum_{i=1}^{m} C_{i} e^{-s \tau_{i}}\right]=0
$$

Obviously, the characteristic equation (57) includes the transcendental terms. It is very difficult that one precisely solves the roots of (57). An application of Theorem 7 yields that the zero solution $x(t) \equiv 0$ of system (1) with (52) is delayindependent globally asymptotically stable.

\section{Conclusions}

In this paper, the delay-independent asymptotic stability of linear fractional-order linear neutral differential systems with multiple discrete delays has been discussed. We have synchronously taken into account the factors of such systems including Caputo's fractional-order derivative, state delays. The asymptotic stability criteria have been derived based on the algebraic approach and matrix theory, which ensure the asymptotic stability for all time-delay parameters. By applying these stability criteria, one can avoid solving the roots of transcendental equations. The results obtained are computationally flexible and efficient. In fact, the characteristic equation of system (1) with (52) includes the transcendental terms. Generally, it is very difficult that one precisely solves the roots of characteristic equation. In Example 1, we analyse the distribution of characteristic roots when the coefficient matrices satisfy the appropriate conditions. We only need to check the spectrum range under conditions $\left(H_{1}\right),\left(H_{4}\right)$, and $\left(H_{5}\right)$. An application of Theorem 7 yields that the zero solution $x(t) \equiv 0$ of system (1) is delay-independent globally asymptotically stable. Example 1 shows that Theorem 7 is computationally flexible and efficient. The stability analysis of linear fractional singular (delay) differential systems will become our future investigative works.

\section{Conflict of Interests}

The authors declare that there is no conflict of interests regarding the publication of this paper.

\section{Acknowledgments}

This paper is dedicated to Professor Zuxiu Zheng on the occasion of his 80th birthday and the 50th anniversary of his research work. The authors are very grateful to the associate editor, Professor Anna Mercaldo, and the two anonymous reviewers for their helpful and valuable comments and suggestions which improved the quality of the paper. This work is supported by the National Natural Science Foundation of China under Grant nos. 11072059 and 61272530, the Specialized Research Fund for the Doctoral Program of Higher Education under Grant nos. 20110092110017 and 20130092110017, the Natural Science Foundation of Jiangsu Province of China under Grant no. BK2012741, and the Programs of Educational Commission of Anhui Province of China under Grant nos. KJ2011A197 and KJ2013Z186.

\section{References}

[1] K. S. Miller and B. Ross, An Introduction to the Fractional Calculus and Fractional Differential Equations, John Wiley \& Sons, New York, NY, USA, 1993.

[2] I. Podlubny, Fractional Differential Equations, vol. 198 of Mathematics in Science and Engineering, Technical University of Kosice, Kosice, Slovak Republic, 1999.

[3] A. A. Kilbas, H. M. Srivastava, and J. J. Trujillo, Theory and Applications of Fractional Differential Equations, vol. 204 of North-Holland Mathematics Studies, Elsevier Science, Amsterdam, The Netherlands, 2006.

[4] K. Diethelm, The Analysis of Fractional Differential Equations: An Application-Oriented Exposition Using Differential Operators of Caputo Type, Springer, Berlin, Germany, 2010.

[5] V. Lakshmikantham, S. Leela, and D. J. Vasundhara, Theory of Fractional Dynamic Systems, Cambridge Scientific Publisher, Cambridge, UK, 2009.

[6] D. Baleanu, J. A. T. Machado, and A. C. J. Luo, Fractional Dynamics and Control, New York, NY, USA, Springer, 2012.

[7] V. Lakshmikantham, "Theory of fractional functional differential equations," Nonlinear Analysis, Theory, Methods and Applications, vol. 69, no. 10, pp. 3337-3343, 2008.

[8] V. Lakshmikantham, "Theory of fractional functional differential equations," Nonlinear Analysis, Theory, Methods \& Applications, vol. 69, no. 10, pp. 3337-3343, 2008.

[9] H. Zhang, J. Cao, and W. Jiang, "General solution of linear fractional neutral differential difference equations," Discrete Dynamics in Nature and Society, vol. 2013, Article ID 489521, 7 pages, 2013.

[10] H. Zhang, J. Cao, and W. Jiang, "Controllability criteria for linear fractional differential systems with state delay and impulses," Journal of Applied Mathematics, vol. 2013, Article ID 146010, 9 pages, 2013.

[11] H. Zhang, J. Cao, and W. Jiang, "Reachability and controllability of fractional singular dynamical systems with control delay," 
Journal of Applied Mathematics, vol. 2013, Article ID 567089, 10 pages, 2013.

[12] R. Sakthivel, P. Revathi, and Y. Ren, "Existence of solutions for nonlinear fractional stochastic differential equations," Nonlinear Analysis, Theory, Methods \& Applications, vol. 81, pp. 70-86, 2013.

[13] J. Cao and L. Wang, "Exponential stability and periodic oscillatory solution in BAM networks with delays," IEEE Transactions on Neural Networks, vol. 13, no. 2, pp. 457-463, 2002.

[14] J. Cao, K. Yuan, and H. X. Li, "Global asymptotical stability of recurrent neural networks with multiple discrete delays and distributed delays," IEEE Transactions on Neural Networks, vol. 17, no. 6, pp. 1646-1651, 2006.

[15] G. Hu, G. Hu, and X. Zou, "Stability of linear neutral systems with multiple delays: boundary criteria," Applied Mathematics and Computation, vol. 148, no. 3, pp. 707-715, 2004.

[16] J. Kuang, H. Tian, and T. Mitsui, "Asymptotic and numerical stability of systems of neutral differential equations with many delays," Journal of Computational and Applied Mathematics, vol. 223, no. 2, pp. 614-625, 2009.

[17] J. Kuang, H. Tian, and K. Shan, "Asymptotic stability of neutral differential systems with many delays," Applied Mathematics and Computation, vol. 217, no. 24, pp. 10087-10094, 2011.

[18] E. Braverman and B. Karpuz, "Uniform exponential stability of first-order dynamic equations with several delays," Applied Mathematics and Computation, vol. 218, no. 21, pp. 10468-10485, 2012.

[19] Z. Orman and S. Arik, "An analysis of stability of a class of neutral-type neural networks with discrete time delays," Abstract and Applied Analysis, vol. 2013, Article ID 143585, 9 pages, 2013.

[20] D. Wu, H. Zhang, J. Cao, and T. Hayat, "Stability and bifurcation analysis of a nonlinear discrete logistic model with delay," Discrete Dynamics in Nature and Society, vol. 2013, Article ID 463059, 7 pages, 2013.

[21] R. Sakthivel and Y. Ren, "Exponential stability of second-order stochastic evolution equations with Poisson jumps," Communications in Nonlinear Science and Numerical Simulation, vol. 17, no. 12, pp. 4517-4523, 2012.

[22] Y. Ren and R. Sakthivel, "Existence, uniqueness, and stability of mild solutions for second-order neutral stochastic evolution equations with infinite delay and Poisson jumps," Journal of Mathematical Physics, vol. 53, no. 7, Article ID 073517, 2012.

[23] D. Matignon, "Stability result on fractional differential equations with applications to control processing," in Proceedings of the International Meeting on Automated Compliance Systems and the International Conference on Systems, Man, and Cybernetics (IMACS-SMC '96), pp. 963-968, Lille, France, 1996.

[24] D. Matignon, "Stability properties for generalized fractional differential systems," Proceedings of the Fractional Differential Systems: Models, Methods and Applications, vol. 5, pp. 145-158, 1998.

[25] Y. Li, Y. Chen, and I. Podlubny, "Mittag-Leffler stability of fractional order nonlinear dynamic systems," Automatica, vol. 45, no. 8, pp. 1965-1969, 2009.

[26] Z. Wang, D. Yang, T. Ma, and N. Sun, "Stability analysis for nonlinear fractional-order systems based on comparison principle," Nonlinear Dynamics, vol. 75, no. 1-2, pp. 387-402, 2014.
[27] Y. Li, Y. Chen, and I. Podlubny, "Stability of fractional-order nonlinear dynamic systems: Lyapunov direct method and generalized Mittag-Leffler stability," Computers and Mathematics with Applications, vol. 59, no. 5, pp. 1810-1821, 2010.

[28] J. Sabatier, M. Moze, and C. Farges, "LMI stability conditions for fractional order systems," Computers and Mathematics with Applications, vol. 59, no. 5, pp. 1594-1609, 2010.

[29] J. V. Devi, F. A. M. Rae, and Z. Drici, "Variational Lyapunov method for fractional differential equations," Computers \& Mathematics with Applications, vol. 64, no. 10, pp. 2982-2989, 2012.

[30] J. R. Wang, L. L. Lv, and Y. Zhou, "New concepts and results in stability of fractional differential equations," Communications in Nonlinear Science and Numerical Simulation, vol. 17, no. 6, pp. 2530-2538, 2012.

[31] M. Rivero, S. V. Rogosin, J. A. Tenreiro Machado, and J. J. Trujillo, "Stability of fractional order systems," Mathematical Problems in Engineering, vol. 2013, Article ID 356215, 14 pages, 2013.

[32] C. P. Li and F. R. Zhang, "A survey on the stability of fractional differential equations," European Physical Journal: Special Topics, vol. 193, no. 1, pp. 27-47, 2011.

[33] S. K. Choi, B. Kang, and N. Koo, "Stability for Caputo fractional differential systems," Abstract and Applied Analysis, vol. 2014, Article ID 631419, 6 pages, 2014.

[34] X. Zhang, "Some results of linear fractional order time-delay system," Applied Mathematics and Computation, vol. 197, no. 1, pp. 407-411, 2008.

[35] M. P. Lazarević and A. M. Spasić, "Finite-time stability analysis of fractional order time-delay systems: gronwall's approach," Mathematical and Computer Modelling, vol. 49, no. 3-4, pp. 475481, 2009.

[36] P. Denghao and J. Wei, "Finite-time stability of neutral fractional time-delay systems via generalized Gronwalls inequality," Abstract and Applied Analysis, vol. 2014, Article ID 610547, 4 pages, 2014.

[37] W. Deng, C. Li, and J. Lu, "Stability analysis of linear fractional differential system with multiple time delays," Nonlinear Dynamics, vol. 48, no. 4, pp. 409-416, 2007.

[38] S. J. Sadati, D. Baleanu, A. Ranjbar, R. Ghaderi, and T. Abdeljawad, "Mittag-Leffler stability theorem for fractional nonlinear systems with delay," Abstract and Applied Analysis, vol. 2010, Article ID 108651, 7 pages, 2010.

[39] D. Baleanu, S. J. Sadati, R. Ghaderi, A. Ranjbar, T. Abdeljawad, and F. Jarad, "Razumikhin stability theorem for fractional systems with delay," Abstract and Applied Analysis, vol. 2010, Article ID 124812, 9 pages, 2010.

[40] E. Kaslik and S. Sivasundaram, "Analytical and numerical methods for the stability analysis of linear fractional delay differential equations," Journal of Computational and Applied Mathematics, vol. 236, no. 16, pp. 4027-4041, 2012.

[41] M. de la Sen, "About robust stability of Caputo linear fractional dynamic systems with time delays through fixed point theory," Fixed Point Theory and Applications, vol. 2011, Article ID 867932, 19 pages, 2011.

[42] Y. Li and Y. Wang, "Uniform asymptotic stability of solutions of fractional functional differential equations," Abstract and Applied Analysis, vol. 2013, Article ID 532589, 8 pages, 2013. 
[43] R. Sakthivel, P. Revathi, and N. I. Mahmudov, "Asymptotic stability of fractional stochastic neutral differential equations with infinite delays," Abstract and Applied Analysis, vol. 2013, Article ID 769257, 9 pages, 2013.

[44] H. Zhang, D. Wu, and J. Cao, "Stability analysis for fractionalorder linear singular delay differential Systems," Discrete Dynamics in Nature and Society, vol. 2014, Article ID 850279, 8 pages, 2014.

[45] J. Chen, K. H. Lundberg, D. E. Davison, and D. S. Bernstein, "The final value theorem revisited: infinite limits and irrational functions," IEEE Control Systems Magazine, vol. 27, no. 3, pp. 97-99, 2007. 


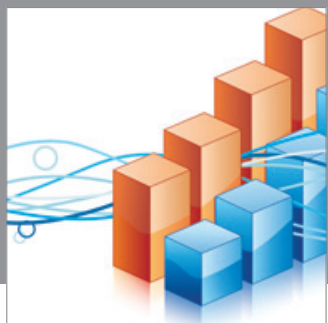

Advances in

Operations Research

mansans

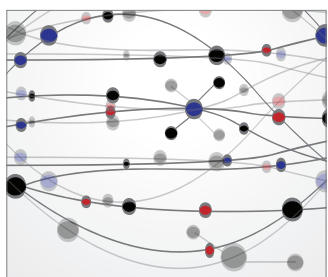

The Scientific World Journal
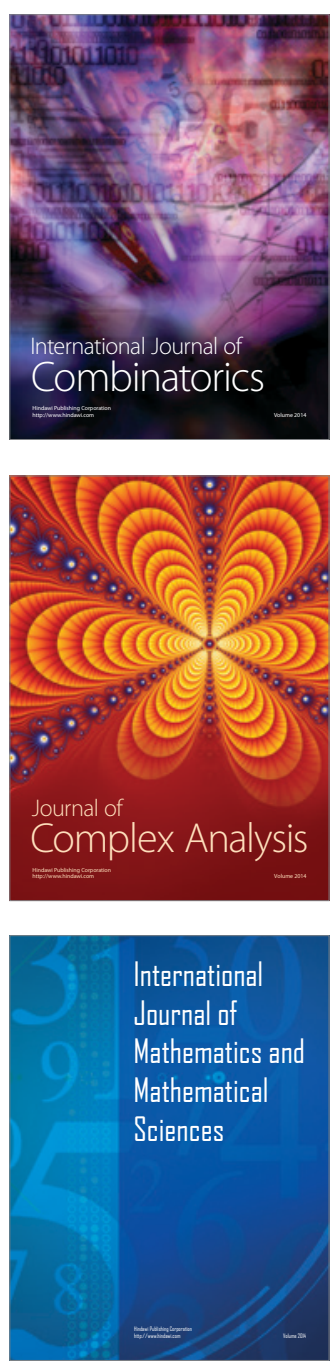
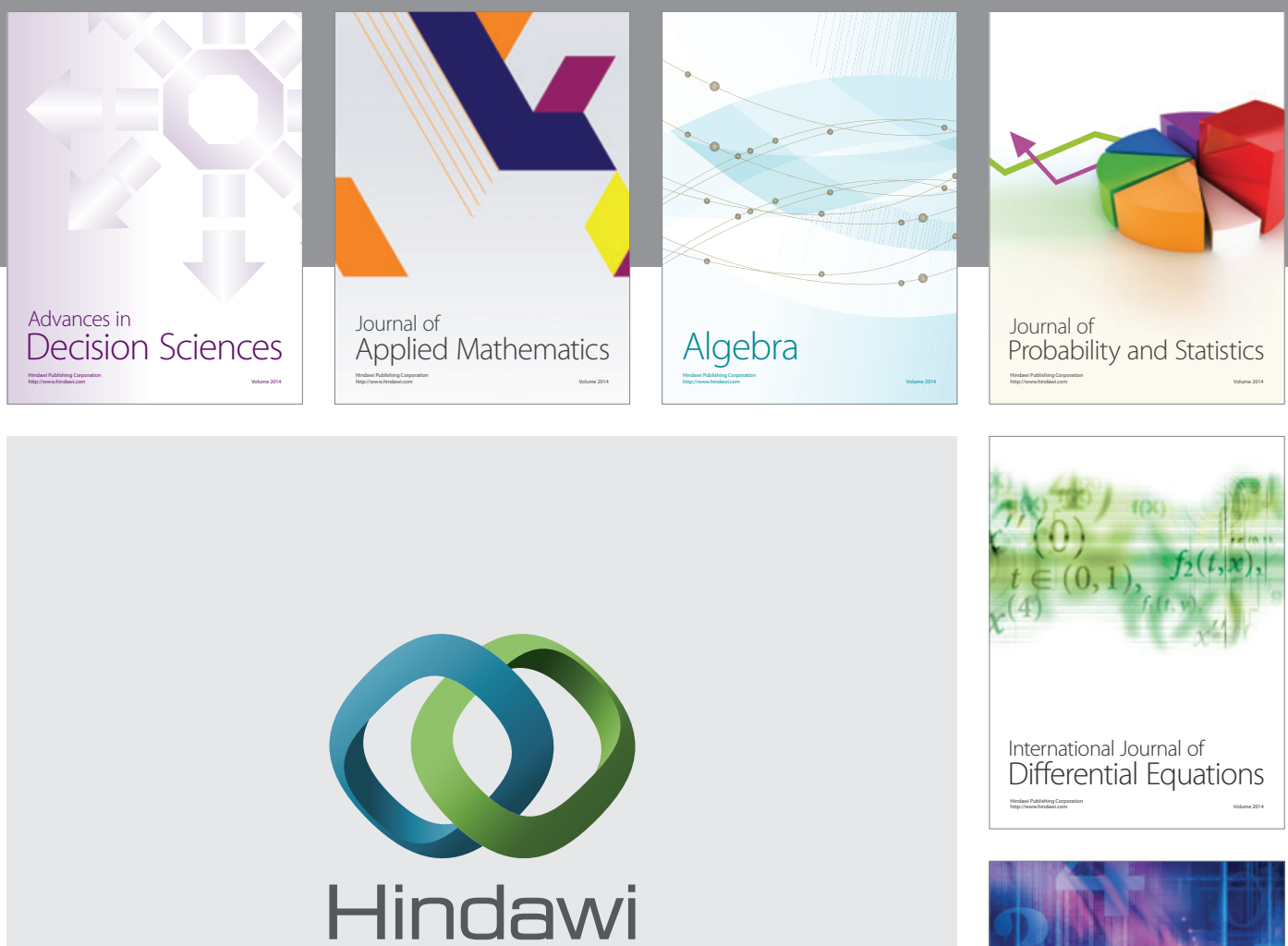

Submit your manuscripts at http://www.hindawi.com
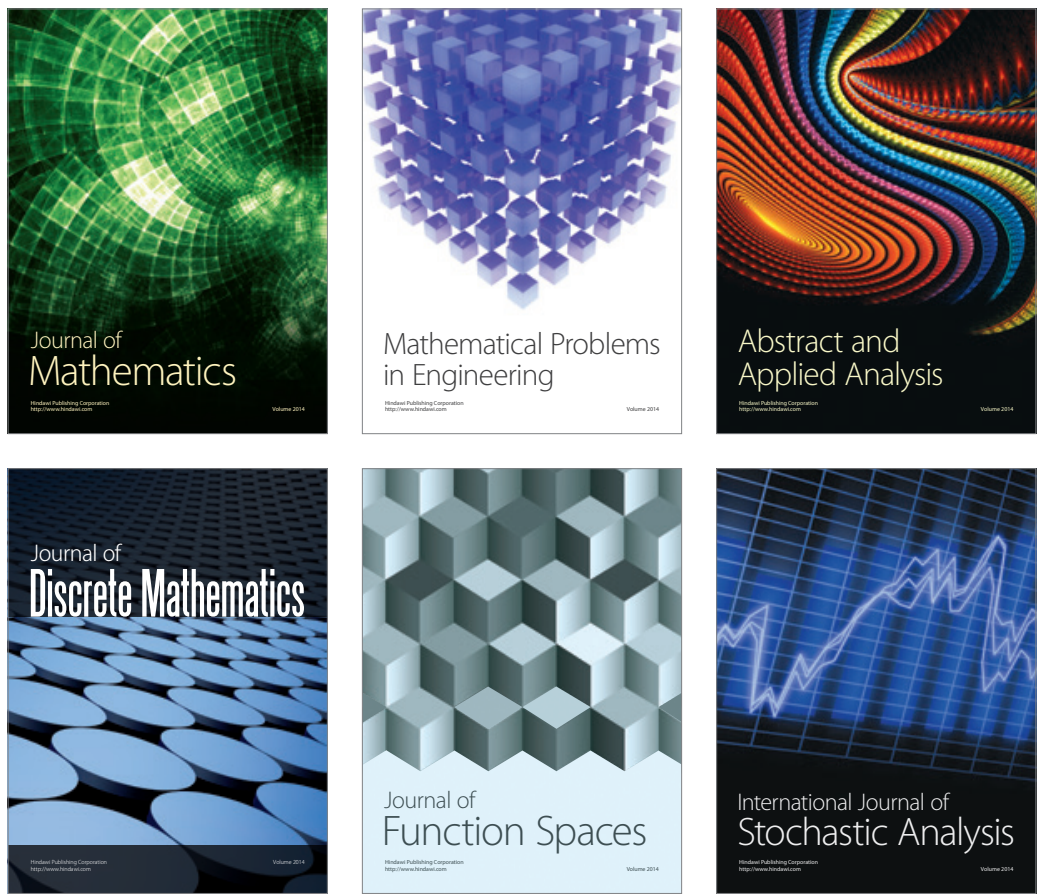

Journal of

Function Spaces

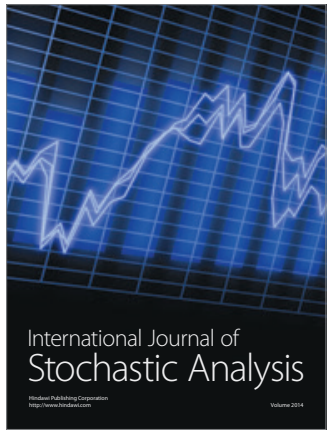

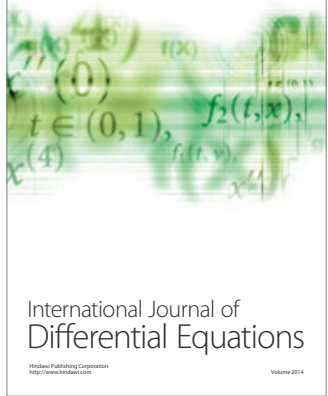
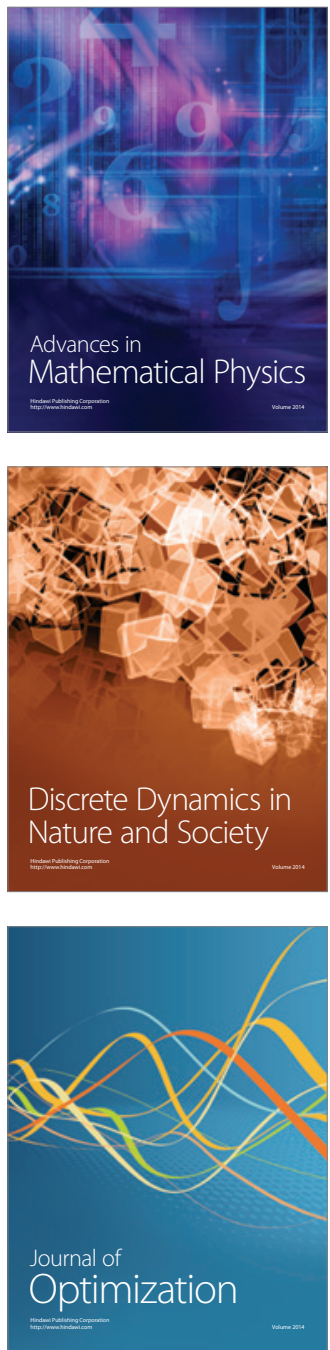